\title{
Sphl RFLP at the human growth hormone gene cluster
}

\section{Gerardo Jiménez ${ }^{1,2}$, Anthony M.Ford ${ }^{1}$ and \\ Albert Boronat ${ }^{2}$ \\ ${ }^{1}$ Leukaemia Research Fund Centre, Institute of Cancer \\ Research, Chester Beatty Laboratories, 237 Fulham \\ Road, London SW3 6JB, UK and 2 Unidad de Bioquímica y Biología Molecular, Facultad de Química, Universidad de Barcelona, 08028 Barcelona, Spain}

Source/Description: The probe used was an EcoRI-HindIII fragment (position 36414-36587) of the human growth hormone (hGH) locus (filled rectangle in Fig. 1). Duplications of this sequence are also present at positions 21632-21804 and 59843-60016 of the locus (unfilled rectangles in Fig.1) (1).

Polymorphism: SphI detects a two allele polymorphism with bands of $>23(\mathrm{~J} 1)$ and $4.1 \mathrm{~kb}(\mathrm{~J} 2)$. In addition, two constant bands of 6.0 and $>23 \mathrm{~kb}$ are observed.

Frequency: Estimated in 24 unrelated individuals.

$\mathrm{J} 1: 0.65$

J2: 0.35

Chromosomal Localization: 17q22-24 (1).

Mendelian Inheritance: Confirmed in two informative families.

Probe Availability: Contact G.J.

Other Comments: RFLPs for BamHI, BglII, HincII and MspI have been previously identified at the $\mathrm{hGH}$ locus (2). The actual size of the $\mathrm{J} 1$ allele is $31.6 \mathrm{~kb}$ (1). The $>23 \mathrm{~kb}$ constant band is a heterogeneous fragment which is $27.5 \mathrm{~kb}$ in size in the $\mathrm{J} 2$ haplotype and corresponds to the $\mathrm{J} 1$ allele in the $\mathrm{J} 1$ haplotype. In the heterozygote, the $>23 \mathrm{~kb}$ band is a mixture of both the 27.5 and the $31.6 \mathrm{~kb}$ fragments.

Acknowledgements: We are grateful to E.Gardner and Dr.B.Ponder for providing family samples. This work was partly supported by the Leukaemia Research Fund (UK). G.J. is the recipient of a predoctoral fellowship from the MEC (Spain).

References: 1) Chen,E.Y. et al. (1989) Genomics 4, 479-497. 2) Chakravarti,A. et al. (1984) Proc. Natl. Acad. Sci. USA 81, 6085-6089.

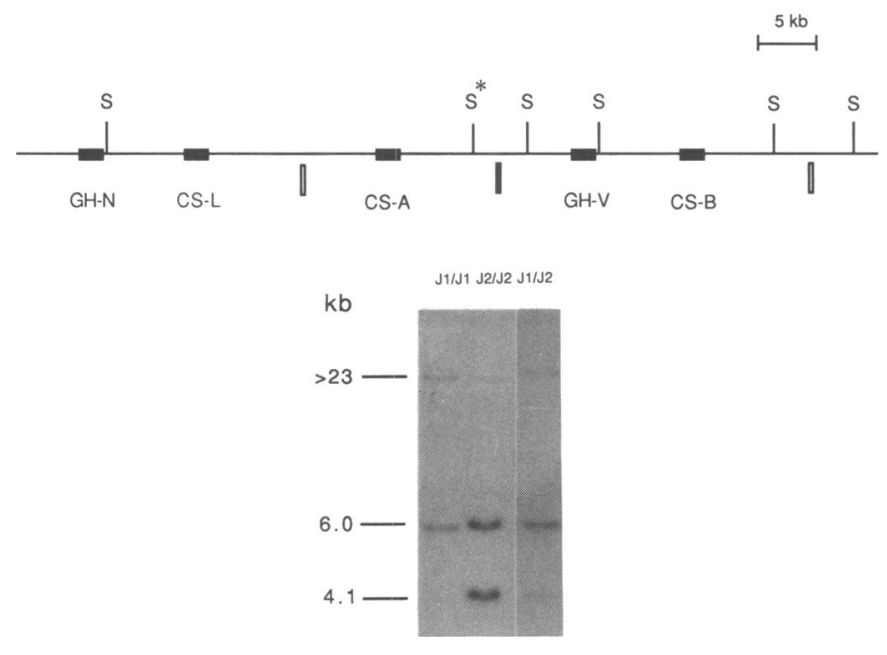

Dinucleotide repeat polymorphism at the PGK1P1 locus

D.L.Browne, J.Zonana and M.Litt*

Oregon Health Sciences University, Portland, OR 97201, USA

Source and Description of Clone: The $(\mathrm{CA})_{14}$ repeat region in cosmid ICRFc100F0599 (EMBL accession number X56251) has been previously described (1).

PCR Primers:

PGK/5A = 5'-TCTACATGTACTTAACCTGC-3'

$\mathrm{PGK} / 5 \mathrm{~B}=5^{\prime}$-AACTCAGTTTTGAGCTCCTA-3'

Polymorphism, Frequencies, Mendelian Inheritance and PCR Conditions: See reference (1).

Chromosomal Localization: We originally placed this repeat at the PGK1 locus at Xq13 (1) because the cosmid ICRFc100F0599 hybridized with the PGK1 probe PSPT-PGK and no recombinants between the $(C A)_{n}$ repeat and the PGK PstI RFLP were seen in 35 phase known meioses in $4 \mathrm{CEPH}$ families. We have now obtained physical and genetic evidence which places ICRFc100F0599 at the closely linked pseudogene locus PGK1P1 at Xq12 (2). 1.) The published pseudogene sequence (3) was used to design PCR primers which specifically amplify this pseudogene sequence. These primers amplified a single fragment from genomic DNA and ICRFc100F0599; the amplified fragment had the predicted size and contained restriction sites predicted from the unpublished sequence. 2.) Two individuals from different families affected with X-linked hypohydrotic ectodermal dysplasia had recombinant PGK1P1 (CA) $)_{\mathbf{n}}$ PGK PstI RFLP haplotypes.

Acknowledgements: This work was supported by grant HG00022 from the National Institutes of Health. We thank Hans Lehrach for cosmid ICRFc100F0599.

References: 1) Browne,D.L., Zonana,J. and Litt,M. (1991) Nucl. Acids Res. 19, 1721. 2) Mandel,J.-L, Willard,H.F., Nussbaum,R.L., Romeo,G., Puck,J.M. and Davies,K.E. (1989) Cytogenet. Cell Genet. 51, 384-437. 3) Michelson,A.M., Bruns,G.A.P., Morton,C.C. and Orkin,S.H. (1985) J. Biol. Chem. 260, 6982-6992. 